\title{
DEZ ANOS DA EDUCAÇÃO PERMANENTE COMO POLÍTICA DE FORMAÇÃO EM SAÚDE NO BRASIL: UM ESTUDO DAS TESES E DISSERTAÇÕES
}

\author{
TEN YEARS OF PERMANENT EDUCATION AS A HEALTH EDUCATION POLICY IN BRAZIL: A STUDY OF \\ THESES AND DISSERTATIONS
}

\section{DIEZ AÑOS DE LA EDUCACIÓN PERMANENTE COMO POLÍTICA DE FORMACIÓN EN SALUD EN BRASIL: UN ESTUDIO DE LAS TESIS Y DISERTACIONES}

\author{
Eluana Borges Leitão de Figueiredo ${ }^{1}$ \\ Mônica Villela Gouvêa ${ }^{2}$ \\ Elaine Antunes Cortez ${ }^{3}$
}

Resumo $\mathrm{O}$ artigo teve como objetivo revelar a produção do conhecimento sobre educação permanente em saúde com base no levantamento de teses e dissertações brasileiras produzidas desde a publicação da primeira portaria relativa à Política Nacional de Educação Permanente, em 2004, até 2013. Foram pesquisadas informações sobre autoria, conteúdo e achados, avaliados em perspectiva bibliométrica. Constatou-se a predominância na autoria de mulheres com formação em enfermagem e serviço social e filiação institucional nas regiões Sul e Sudeste do Brasil. Verificou-se a concentração de estudos descritivo-exploratórios, abordagens qualitativas e entrevistas semiestruturadas como técnica de coleta de dados. Na análise das referências bibliográficas, observaram-se três categorias: publicações do Ministério da Saúde, referências de autores diversos e referências relacionadas ao método. A pesquisa procurou reforçar a necessidade de inclusão da 'educação permanente' como descritor e revelou concentração de estudos no campo das políticas de saúde, atenção básica e ensino.

Palavras-chave educação permanente em saúde; políticas públicas; bibliometria.

\author{
Silvia Cristina Pereira dos Santos ${ }^{4}$ \\ Kyra Vianna Alóchio ${ }^{5}$ \\ Lauanna Malafaia da Silva Alves ${ }^{6}$
}

\begin{abstract}
The article aimed to reveal the production of knowledge on permanent health education based on a survey of Brazilian theses and dissertations produced since the enactment of the first ordinance on the National Policy for Permanent Education, in 2004 until the year 2013. Information on authorship, content, and findings were surveyed and evaluated from the bibliometric perspective. It was found that there is a predominance of authorship by women with training in nursing and social services and with institutional affiliation in Southern and Southeastern Brazil. It was noted that there is a concentration of descriptive and exploratory studies, qualitative approaches and semi-structured interviews as a data collection technique. When analyzing the bibliographic references, there were three categories: Ministry of Health publications, references from various authors, and references related to the method. The research sought to reinforce the need for the inclusion of 'permanent education' as a descriptor and revealed a concentration of studies in the fields of health policy, primary health care, and education.
\end{abstract}

Keywords permanent education in health; public policies; bibliometrics. 


\section{Introdução}

A Política Nacional de Educação Permanente em Saúde (PNEPS), instituída em 2004 por meio da portaria n. 198, de 13 de fevereiro, e posteriormente substituída pela portaria GM/MS n. 1.996, de 20 de agosto de 2007, constitui um importante marco na implementação de diretrizes educacionais nos diferentes processos de trabalho em saúde (Brasil, 2004, 2007). A educação permanente em saúde (EPS) é referida na PNEPS como uma proposta política e pedagógica baseada na aprendizagem significativa e na perspectiva de transformação das práticas profissionais. No Brasil, a EPS se configurou como política pública diante da necessidade de se promoverem práticas ancoradas no contexto real dos serviços, dos trabalhadores e das necessidades dos usuários (Brasil, 2004, 2007).

O Programa de Mestrado Profissional em Ensino na Saúde da Universidade Federal Fluminense (MPES/UFF) tem uma linha de pesquisa voltada para a educação permanente em saúde no Sistema Único de Saúde (SUS). Nessa linha, o presente estudo derivou de inquietações de docentes e profissionais de saúde com relação à carência de sistematização do conhecimento produzido desde a publicação da primeira portaria da PNEPS no Brasil, em 2004 (Brasil, 2004). Dessa forma, ao se partir do pressuposto de que a literatura é o ingrediente principal no processo de comunicação do conhecimento e que a análise da produção científica é sempre relevante em qualquer campo de atuação, o artigo pretendeu realizar uma análise bibliométrica da produção científica de teses e dissertações sobre a EPS desde a primeira publicação da PNEPS, em 2004.

\section{Caminhos da pesquisa}

A pesquisa que deu origem a este artigo foi do tipo descritiva, com abordagem quantitativa à luz da técnica bibliométrica, referida por Araújo (2006, p. 12) como uma "técnica quantitativa e estatística de medição dos índices de produção e disseminação do conhecimento científico".

Neste estudo, optou-se pelo uso combinado de técnicas bibliométricas descritivas e avaliativas com base em classificação de Narin (1976). Segundo o autor, a bibliometria descritiva decorre do estudo de características tais como a distribuição geográfica e a evolução temporal, enquanto a bibliometria avaliativa volta-se para aspectos qualitativos da atividade científica. No entanto, cumpre ressaltar que a bibliometria sofre críticas, algumas delas referentes à ênfase em dados quantitativos que não abarcam contextos e fatores históricos, inviabilizando, assim, outros tipos de análises. Alerta-se 
também para o fato de que se trata de uma abordagem que favorece a observação dos meios em detrimento dos fins na avaliação da produção científica (Alvarenga, 1998).

O levantamento se deu em duas etapas e pode ser visualizado na Figura 1. A etapa 1 envolveu a busca na base de dados da Coordenação de Aperfeiçoamento de Pessoal de Nível Superior (Capes), uma vez que esta é considerada uma base importante de dissertações e teses. Nessa fase, foram incluídas todas as dissertações e teses relacionadas à EPS, de universidades públicas e privadas, e excluídos os estudos duplicados, que não estivessem disponíveis na íntegra e que envolvessem educação permanente em outras áreas de conhecimento que não a saúde.

A etapa 2 foi realizada com o mesmo intervalo temporal da busca na base Capes (2004-2013), em bancos de dados de universidades públicas federais e estaduais. A busca objetivou localizar nos bancos específicos das instituições de ensino estudos que não tivessem sido publicados pela Capes, de forma a sanar o déficit de trabalhos relativos ao ano de 2013 ainda não disponíveis na primeira base consultada. A busca envolveu análise de todas as universidades públicas pertencentes às esferas estaduais e federais do Brasil, nas regiões Norte, Sul, Sudeste, Centro-Oeste e Nordeste.

Foram incluídas, no estudo, as bases de acesso livre e disponíveis no acervo sem necessidade de construção de senhas ou logins e com resposta positiva do servidor às solicitações de busca, em tempo menor do que dois minutos de espera. Nessa etapa, excluíram-se trabalhos que não versavam sobre educação permanente na área da saúde, trabalhos não disponíveis para download na íntegra e trabalhos já identificados na primeira etapa.

A consulta às bases de dados foi realizada entre os meses de agosto e dezembro de 2013. Procedeu-se à busca por todos os estudos publicados no intervalo de tempo de 2004 a 2013, utilizando-se as seguintes palavras-chave: 'educação permanente em saúde (EPS)' e 'educação permanente (EP)' na ferramenta de busca e consulta de resumos, uma vez que não existem descritores. O uso da segunda expressão se deveu ao fato de que muitos estudos abordavam a temática dessa forma, apesar de tratarem de educação permanente no campo da saúde. Ressalte-se que, durante o período da coleta de dados, tanto a Capes como as universidades públicas brasileiras disponibilizavam as publicações em suas bases até dezembro de 2012 .

Após a conclusão das duas etapas, procedeu-se ao cruzamento dos dados das planilhas geradas, procurando-se eliminar possíveis duplicações. Os documentos na íntegra foram salvos em arquivo digital, com numeração específica correspondente ao identificador do quadro final da revisão.

Os 54 estudos foram analisados e classificados conforme os seguintes indicadores bibliométricos: relacionados à autoria - gênero, formação e 
região/filiação institucional; e relacionados ao conteúdo - descritores e palavras-chave utilizados, temáticas relacionadas, explicitação de referenciais bibliográficos (EPS e métodos) e método (tipo de estudo, abordagem e técnicas).

Figura 1

Esquema gráfico dos caminhos da busca bibliográfica

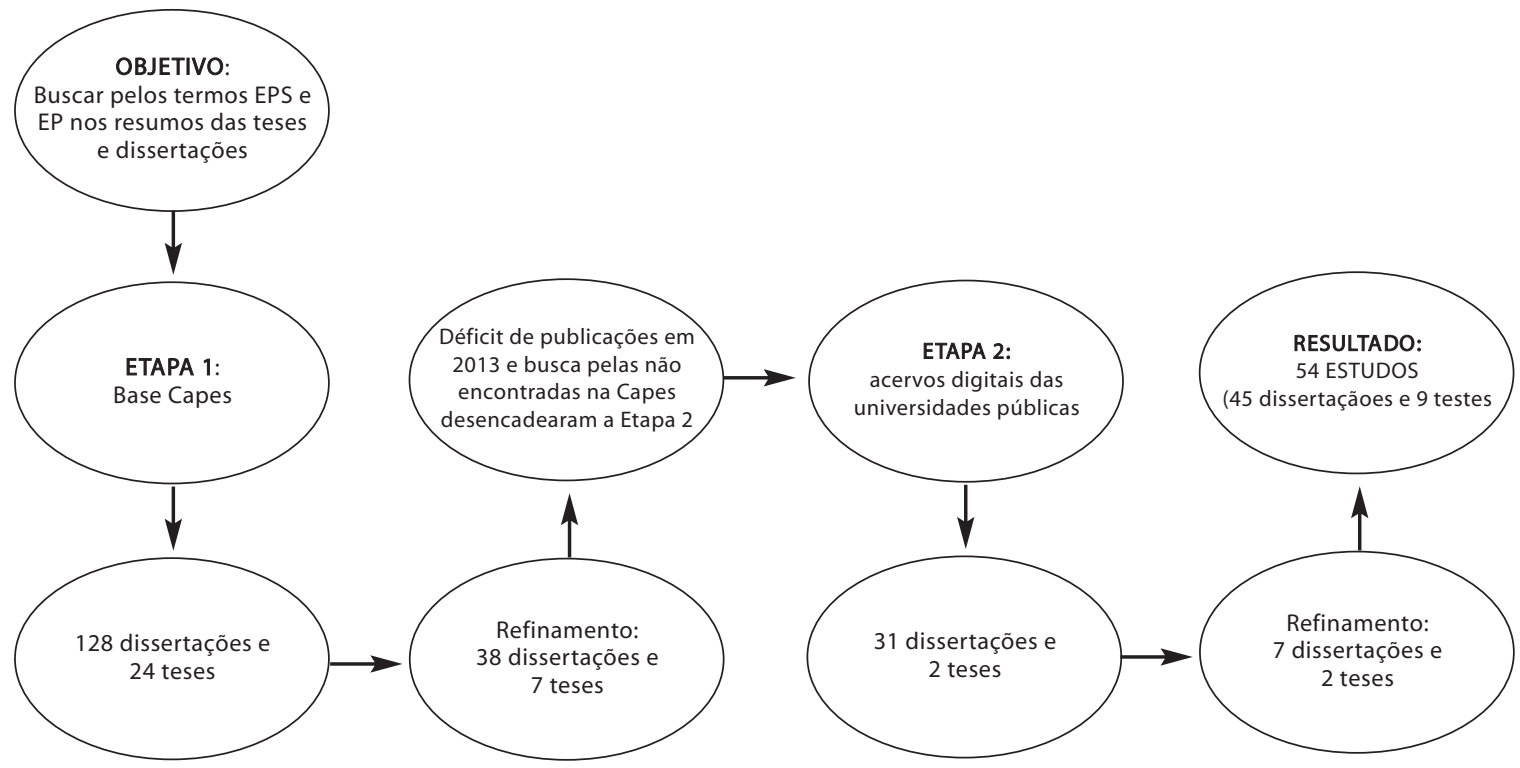

Fonte: As autoras.

\section{Publicações em EPS pós-PNEPS: teses e dissertações}

Os trabalhos selecionados a partir da base Capes representaram $83 \%$ dos estudos incluídos na pesquisa. Os restantes $17 \%$ foram oriundos da busca nos portais das universidades (etapa 2). A busca realizada na primeira etapa da pesquisa revelou ausência de teses e dissertações publicadas no ano de 2013 sobre a temática pesquisada; assim, optou-se, numa segunda etapa, por identificar possíveis estudos mais recentes e ainda não disponíveis na base Capes. Procedeu-se, então, à busca por dissertações e teses nos acervos digitais de universidades públicas situadas nas diversas regiões brasileiras.

Foram pesquisadas 102 universidades que tinham bases de dados disponíveis (64 federais e 38 estaduais): oito na região Centro-Oeste; 15 na Norte; 32 na Nordeste; 27 na Sudeste; e vinte na Sul. 
Ressalte-se que, na busca feita no banco de dados das universidades, foram encontradas duas teses de doutorado e sete dissertações de mestrado na temática de EPS e EP, sendo duas dissertações de 2013. Não foi possível identificar, nos estudos analisados ou por pesquisa no currículo Lattes dos autores, se as dissertações encontradas nas fases 1 e 2 pertenciam a mestrados acadêmicos ou profissionais.

Dentre os 54 trabalhos selecionados para o estudo, verificou-se uma concentração de dissertações $(83 \%)$ em detrimento de teses $(17 \%)$. O termo 'educação permanente' apareceu em 76\% dos títulos: 25 dissertações e cinco teses; já o termo 'educação permanente em saúde' compôs outros $24 \%$ : 17 dissertações e quatro teses. A análise do número de publicações mostrou o quanto foi publicado em termos de EPS no Brasil, desde a implantação da PNEPS até 2013.

O Gráfico 1 ilustra a evolução ano a ano do número de teses e dissertações publicadas no período de 2004 a 2013. Observou-se uma primeira dissertação publicada em 2004 e pequenos picos de produção nos anos de 2008, 2010 e 2012. As primeiras teses foram publicadas em 2007 e 2008, respectivamente, decrescendo para uma publicação por ano a partir de 2011.

\section{Gráfico 1}

Evolução das publicações de teses e dissertações por ano no período de estudo

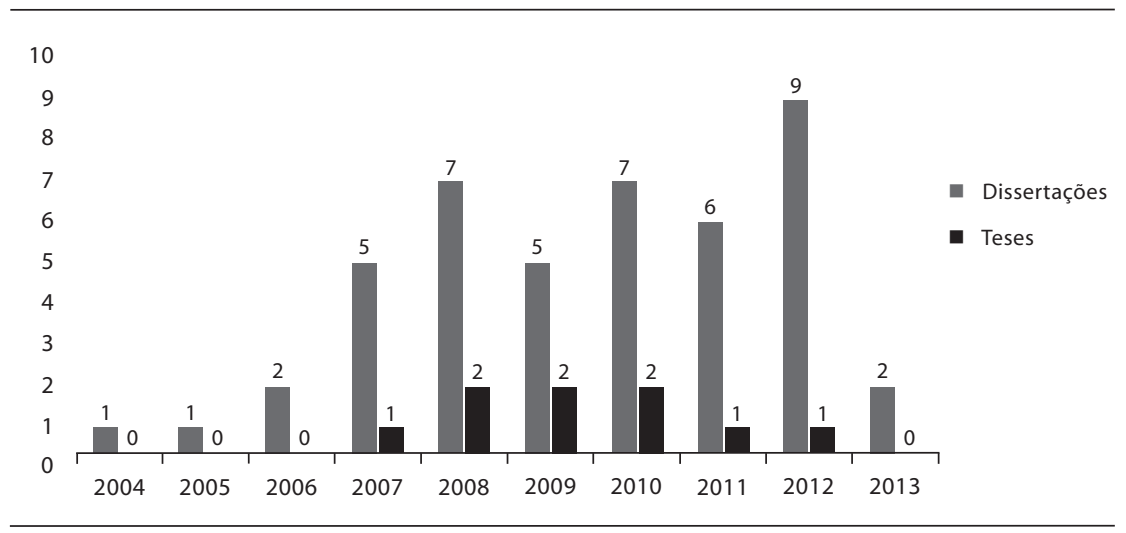

Fonte: As autoras.

\section{Autoria de teses e dissertações}

Ao se observar a autoria das publicações segundo o gênero, verificou-se que a grande maioria dos autores (teses e dissertações) sobre a temática da EPS no período investigado era do gênero feminino (91\%). O resultado se repetiu quando se analisaram as publicações separadamente: dentre as 45 dissertações, $91 \%$ eram de autores femininos e $9 \%$ de masculinos; e das 
nove teses, $58 \%$ apresentaram autores do gênero feminino em relação a $42 \%$ do gênero masculino.

A formação dos autores foi pesquisada por meio da busca de currículos na Plataforma Lattes, uma vez que essa informação não constava na maioria dos documentos reunidos. Com relação às dissertações, dentre os 45 autores foram encontrados 21 enfermeiros, cinco assistentes sociais, três odontólogos, dois médicos, três farmacêuticos, um psicólogo, um comunicador social, um administrador, um nutricionista e um terapeuta ocupacional. Quatro nomes não foram encontrados na Plataforma Lattes, e não foi possível identificar a formação de seis autores.

Na busca por autores de teses sobre o tema EPS, encontraram-se três assistentes sociais, dois médicos, dois enfermeiros, um odontólogo e um sociólogo. O estudo revelou uma expressiva participação de enfermeiros (47\%) na autoria de teses e dissertações no período investigado, seguida por assistentes sociais (17\%). No perfil de trabalho investigado, observou-se um tímido interesse de outras profissões na produção de conhecimento sobre a EPS.

Quanto à procedência geográfica, a pesquisa evidenciou uma disparidade regional em relação à publicação sobre o tema. As dissertações e teses sobre EPS se apresentaram, prioritariamente, na regiões Sudeste $(44,4 \%)$ e Sul $(35 \%)$, seguindo-se as do Nordeste $(12,5 \%)$ e do Centro-Oeste $(9 \%)$. A região Norte não produziu nenhum trabalho científico de pós-graduação stricto sensu sobre o tema no período do estudo.

Ao se detalhar a filiação institucional, observou-se que dentre as universidades sediadas na região Sudeste destacou-se o Rio de Janeiro, com dez dissertações. A Universidade do Estado do Rio de Janeiro (Uerj) e a Fundação Oswaldo Cruz (Fiocruz) contribuíram com quatro estudos cada, e a Universidade Federal Fluminense (UFF), com dois. Na sequência, apareceu o estado de Minas Gerais, com cinco trabalhos, tendo contribuído a Universidade Federal de Minas Gerais (UFMG), com duas dissertações e duas teses, e a Universidade Estadual de Montes Claros (UEMC), com um estudo. O estado de São Paulo apareceu com quatro dissertações: duas da Universidade de São Paulo (USP), uma da Universidade Federal de São Carlos (Ufscar) e uma da Universidade Estadual de Campinas (Unicamp); e também com três teses: duas da Universidade Estadual Paulista-Franca/Marília (Unesp) e uma da Universidade de São Paulo (USP). Por fim, apareceu o Espírito Santo, com uma dissertação produzida na Universidade Federal do Espírito Santo (Ufes).

Na região Sul, destacou-se o estado do Rio Grande do Sul, com dez estudos. Quatro eram dissertações da Universidade Federal do Rio Grande (UFRG), uma da Universidade Federal de Pelotas (UFPEL) e uma tese e três 
dissertações da Universidade Federal do Rio Grande do Sul (UFRGS), além da Pontifícia Universidade Católica do Rio Grande do Sul (PUCRS) - única universidade privada com trabalho publicado sobre o tema EPS no portal Capes, com uma publicação. O estado de Santa Catarina apareceu com seis dissertações e uma tese oriundas da Universidade Federal de Santa Catarina (UFSC); na sequência, veio o Paraná, com três dissertações, uma produzida na Universidade Estadual de Londrina (UEL) e duas na Universidade Federal do Paraná (UFPR).

A região Nordeste produziu seis dissertações, oriundas dos estados do Ceará, Bahia, Rio Grande do Norte e Sergipe. Duas eram da Universidade Estadual do Ceará (UECE), duas da Universidade Federal da Bahia (UFBA), uma da Universidade Federal do Rio Grande do Norte (UFRN) e uma da Universidade Federal de Sergipe (UFS).

Por fim, a região Centro-Oeste produziu quatro dissertações, com destaque para a Universidade de Brasília (UnB), com duas publicações, seguindo-se o estado de Goiás, com um trabalho da Universidade Federal de Goiás (UFG), e o estado do Mato Grosso, com um estudo da Universidade Federal de Mato Grosso (UFMT). Foi publicada uma tese pela Universidade Federal de Goiás (UFG).

Não foram encontradas publicações de teses sobre EPS na região Nordeste e teses/dissertações na região Norte do Brasil.

Os resultados permitiram observar as universidades que mais publicaram teses e dissertações sobre EPS no período de estudo: UFSC, UFRGS e UFRG (região Sul) e Uerj, Fiocruz e UFMG (região Sudeste). Tais resultados podem expressar uma tendência regional de reflexão sobre o tema. Enquanto as regiões Sudeste e Sul concentravam 79,4\% das publicações, as regiões Nordeste, Centro-Oeste e Norte, juntas, correspondiam a 20,6\%.

\section{Conteúdo de teses e dissertações}

O estudo permitiu relacionar 105 diferentes descritores e palavras-chave (Tabela 1). Dentre os termos mais utilizados pelos autores como palavras-chave, $40 \%$ constam no portal de descritores em ciências da saúde (DeCS). No entanto, as palavras-chave mais diretamente ligadas à EPS, 'educação permanente em saúde' e 'educação permanente', as mais citadas ( $\mathrm{n}=29)$, não foram encontradas no DeCS. Os descritores mais adotados como fonte de informação e recuperação de assuntos da literatura científica sobre EPS foram: 'educação em saúde' (n=11) 'educação continuada' (n=10) e 'educação' (n=09). Demais descritores e palavras-chave foram citados em média duas vezes. 


\section{Tabela 1}

\begin{tabular}{|c|c|c|}
\hline Palavras-chave & $\mathrm{DeCS}$ & Dissertaçöes e teses \\
\hline Aprendizagem & Sim & 2 \\
\hline Aprendizagem baseada em problemas & Sim & 1 \\
\hline Aprendizagem-avaliação & Não & 1 \\
\hline Capacitação & Sim & 2 \\
\hline Capacitação em serviço & Sim & 1 \\
\hline Concepção de educação & Não & 1 \\
\hline Educação & Sim & 9 \\
\hline Educação continuada & Sim & 10 \\
\hline Educação continuada em enfermagem & Sim & 2 \\
\hline Educação e ensino & Não & 1 \\
\hline Educação e Estado & Não & 1 \\
\hline Educação e saúde & Não & 1 \\
\hline Educação em enfermagem & Sim & 4 \\
\hline Educação em saúde & Sim & 11 \\
\hline Educação permanente & Não & 17 \\
\hline Educação permanente em enfermagem & Não & 1 \\
\hline Educação permanente em saúde & Não & 12 \\
\hline Educação profissional em saúde pública & Sim & 3 \\
\hline Formação de recursos humanos & Não & 2 \\
\hline Formação educacional & Não & 2 \\
\hline Formação profissional & Não & 3 \\
\hline Integração ensino e serviço & Não & 1 \\
\hline Política educacional & Não & 1 \\
\hline Política Nacional de Educação Permanente em Saúde & Não & 1 \\
\hline Problematização & Não & 1 \\
\hline
\end{tabular}

Fonte: As autoras.

A pesquisa nos resumos dos trabalhos permitiu também reconhecer os principais cenários e temas com os quais a EPS esteve relacionada (Gráfico 2). A análise mostrou uma tendência à concentração de discussões no campo 
das políticas de saúde $(\mathrm{n}=15)$ e paritariamente nos campos do ensino/formação (n=l1) e da atenção básica $(n=11)$.

Gráfico 2

Evolução das publicações de teses e dissertações por ano no período de estudo

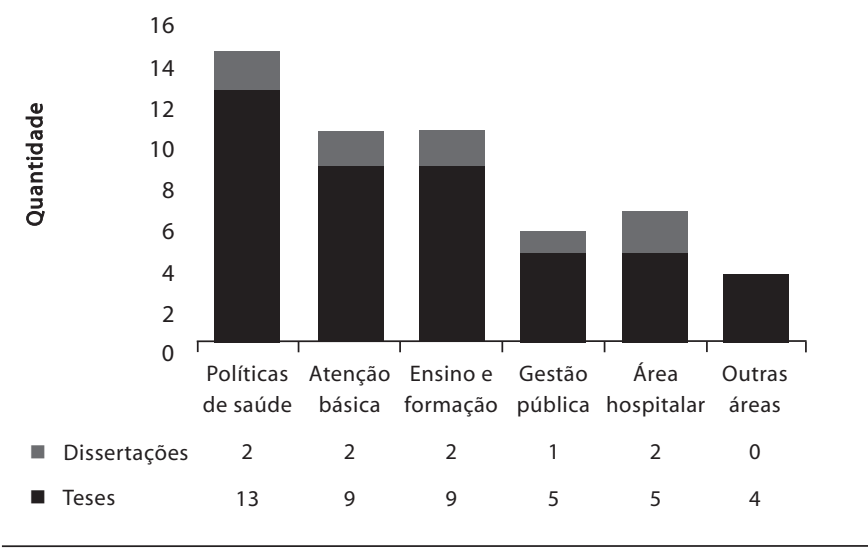

Fonte: As autoras.

Procurou-se analisar se as dissertações e teses examinadas explicitavam um referencial teórico. Tal referencial foi buscado nas seguintes partes do trabalho: resumo, sumário, introdução, método e demais capítulos. Observou-se que em $76 \%$ das dissertações e $50 \%$ das teses o referencial teórico não estava explicitado no texto do trabalho.

$\mathrm{Na}$ análise dos principais métodos adotados em dissertações e teses analisadas, optou-se por identificar três aspectos: tipo, abordagem e principais técnicas de coleta de dados. A maioria $(68,7 \%)$ se apresentou como não experimental (pesquisas descritivas e exploratórias), das quais 23\% não explicitaram com clareza o tipo de estudo. A abordagem qualitativa foi utilizada em 79,6\% dos estudos analisados. A técnica de coleta de dados mais adotada foi a entrevista semiestruturada $(n=23)$.

A análise das referências bibliográficas adotadas nos estudos permitiu classificá-las em três grupos: referências bibliográficas relacionadas a publicações dos ministérios da Saúde ou Educação; referências de autores diversos que publicam sobre EPS; e referências bibliográficas relacionadas ao método adotado.

As referências bibliográficas relacionadas a publicações do Ministério da Saúde (MS) foram citadas cinquenta vezes nas teses e 181 nas dissertações. Os documentos do MS mais citados foram os relacionados à PNEPS $(\mathrm{n}=64)$, aos projetos, programas e relatórios do MS ( $\mathrm{n}=66)$ e aos cadernos de recursos humanos da saúde $(\mathrm{n}=21)$. 
Os autores que abordam o tema EPS mais citados nas dissertações e teses pesquisadas foram: Ricardo Burg Ceccim $(\mathrm{n}=86)$; Maria Cristina Davini ( $\mathrm{n}=43)$; Maria Alice Roschke $(\mathrm{n}=34)$; José Haddad $(\mathrm{n}=36)$ e Emerson Elias Merhy ( $\mathrm{n}=38)$. Os autores mais adotados para a fundamentação bibliográfica dos métodos de pesquisa nas dissertações e teses analisadas foram: Maria Cecília Minayo (n=58); Augusto Triviños $(\mathrm{n}=15)$ e Laurence Bardin (n=14).

\section{Análise da produção de teses e dissertações sobre a EPS}

A investigação acerca da produção de conhecimentos sobre o tema EPS na base de dados Capes e nas universidades públicas brasileiras possibilitou observar que a produção científica de dissertações iniciou-se em 2005, logo após a publicação da PNEPS pela portaria GM/MS n. 198, de 13 de fevereiro de 2004. Entretanto, observou-se que, após 2007, a publicação sobre o tema tanto de dissertações quanto de teses - teve um acréscimo, coincidindo com o ano da publicação da nova PNEPS, instituída pela portaria GM/MS n. 1.996, de 20 de agosto de 2007, que veio dispor sobre novas diretrizes para a implementação da EPS no território brasileiro e alinhar-se às diretrizes do Pacto pela Saúde (Brasil, 2004, 2007).

Esse dado evidenciou que a instituição política da EPS pode ter refletido e impulsionado o aumento da produção científica. Supõe-se que pode ter fomentado o interesse dos mestrados e doutorados na abordagem do tema. Entretanto, ao se considerar a relevância para o setor da saúde, os resultados nos anos que se seguiram à PNEPS mostraram-se discretos e revelaram um campo ainda em crescimento.

Percebeu-se expressiva participação de mulheres na autoria das publicações. O resultado pode ser compreendido a partir dos consideráveis avanços na participação das mulheres no campo científico, em uma ciência com base histórica, predominantemente masculina (Silva e Ribeiro, 2011), como apontam os dados do último censo divulgado pelo Instituto Nacional de Estudos e Pesquisas Educacionais Anísio Teixeira (Inep).

No intervalo temporal deste estudo, 3.223.926 mulheres concluíram cursos superiores no Brasil, em comparação com 2.803.951 homens (Brasil, 2012). O crescimento de mulheres nos cenários educacionais também foi fruto de investimentos do Estado brasileiro na construção da igualdade de gênero, cujo foco concentrou-se na ampliação e promoção dos direitos das mulheres por meio de política específica (Brasil, 2010a). Acredita-se que, além do incremento de mulheres no campo científico, a superioridade do gênero feminino neste estudo pode ser explicada pelo fato de tal discussão ter ocorrido mais entre profissões com maior contingente feminino, como enfermagem e serviço social. 
O estudo apresentou ainda uma significativa produção científica ligada aos temas 'políticas públicas de saúde' e 'atenção básica', o que vem caracterizando marcante presença dessas áreas na produção de conhecimento sobre EPS. Assim, considerando que no Brasil a EPS se constituiu em política de saúde inscrita no campo da educação e formação de trabalhadores em saúde, é compreensível que essas temáticas tenham sido recorrentes nos trabalhos pesquisados.

Da mesma forma, a presença da atenção básica como segunda temática mais relacionada nos estudos se justificou pela importância da atenção primária na discussão da agenda da saúde, uma vez que a opção por um modelo centrado na doença, no hospital e nos procedimentos, que marcou a política nacional até a constituição do SUS, deixou um grande déficit do ponto de vista da garantia de saúde e da formação dos trabalhadores. O modelo de atenção com ênfase na atenção primária como ordenadora das redes de atenção à saúde e coordenadora do cuidado constituiu cenário favorável à discussão sobre práticas transformadoras nos processos de trabalho da equipe de saúde. Assim, é possível que a transição entre os modelos tenha se refletido no âmbito acadêmico e nas produções científicas, em que o diálogo com a realidade e com as práticas profissionais ressignificaram o próprio conhecimento (Leal e Camargo Junior, 2012).

Além da predominância de mulheres e do campo das políticas públicas de saúde nas publicações sobre EPS, os resultados deste estudo mostraram grande representação de autores com formação em enfermagem, que ocuparam o primeiro lugar na autoria. Tal resultado pode estar ligado à expansão de cursos de graduação e pós-graduação no Brasil e ao processo de transformação por que o campo da enfermagem vem passando no sentido da responsabilidade com a formação de recursos humanos para a saúde, bem como o seu papel na produção de conhecimentos científicos (Erdmann, Fernandes e Teixeira, 2011; Vieira et al., 2004). Nesse sentido, o aumento de vagas nos estabelecimentos de ensino superior, segundo Teixeira e colaboradores (2006), vem exigindo cada vez mais a incorporação de novos saberes e indica a necessidade de maior embasamento educacional dos trabalhadores. Talvez isso justifique a escolha desses profissionais pelo tema EPS.

Agregue-se aos dados apresentados a necessidade de incentivo a outros profissionais da saúde na produção de conhecimento sobre a temática, tendo em vista a importância da representação multiprofissional nos arranjos constitutivos da EPS, assim expressos pela política nacional.

As regiões do Brasil com mais publicações sobre o tema EPS também foram analisadas e forneceram indicadores de desigualdades regionais em termos de publicação. A forte presença das regiões Sudeste e Sul nas publicações e a disparidade em relação às outras regiões do país vão ao encontro do Plano Nacional de Pós-Graduação 2011/2020, formulado pela Capes, que 
aponta para uma assimetria na distribuição da pós-graduação no território nacional, principalmente nas regiões Nordeste, Norte e Centro-Oeste. O documento afirma ainda que essas disparidades são homólogas às desigualdades econômicas e sociais prevalentes no Brasil. Nesse sentido, a própria Capes indica no documento que tal assimetria pode ocorrer tanto pela inexistência de instituições de ensino superior em algumas regiões quanto pela inoperância de políticas públicas que favoreçam a igualdade regional (Brasil, 2010b). Assim, o reduzido número de publicações sobre o tema EPS pode não refletir desinteresse regional pelo tema, mas sim uma distribuição desigual da oferta de programas de pós-graduação. Estudo de Rodrigues e colaboradores (2007) revela que somente a região Sudeste concentra 54,9\% dos cursos de mestrado e $66,6 \%$ dos de doutorado; a Sul, 19,6\% e 17, $1 \%$, respectivamente; a Nordeste, 15,6\% e 10,3\%; a Centro-Oeste, 6,4\% e 4,1\%; e a Norte, $3,5 \%$ e $1,8 \%$.

$\mathrm{O}$ resultado apresentado também pode estar relacionado ao fato de que as universidades que mais publicaram sobre o tema, entre as quais UFSC, UFRGS e UFRG (região Sul) e Uerj, Fiocruz e UFMG (região Sudeste), encontram-se nas regiões com maior destaque em publicações. Esse dado pode representar lacunas na comunidade científica e acentuar ainda mais diferenças entre as produções regionais.

A inexistência do descritor 'educação permanente em saúde' no DeCS pode configurar um empobrecimento de publicações nesse campo, devido ao prejuízo no esforço de indexação de assuntos na literatura científica sobre EPS. A necessidade de se encontrarem descritores mais próximos no campo da saúde (sendo em geral eleitos os termos educação continuada e educação em saúde) acabou situando o trabalho em um campo conceitual diferente daquele preconizado pela EPS. Assim, o fato de a maioria dos termos adotados não serem referenciados como um descritor em ciências da saúde pode denotar uma dificuldade na recuperação da informação sobre o assunto EPS nas bases científicas, e o uso de uma terminologia adequada proporcionaria maior eficiência na busca por publicações sobre o tema. De modo geral, relativizamos esse dado ao fator tempo, tendo em vista a EPS ser um tema de publicação recente no universo da educação na saúde e em serviço.

Os estudos apontaram ainda tendências no uso de algumas características metodológicas. A abordagem qualitativa, por exemplo, utilizada em mais de $80 \%$ dos estudos analisados, pode ser oriunda da tendência do campo da saúde em utilizar a abordagem de contextos informais (estudos de caso, observação participante, pesquisa ação e pesquisa intervenção) e temas relacionados a processos educativos e formativos acerca do trabalho, como menciona um estudo sobre o uso da abordagem qualitativa na produção científica em saúde (Vargas et al., 2009). 
$\mathrm{Na}$ análise dos materiais publicados, verificou-se que as referências bibliográficas e metodológicas usadas nos estudos também apresentaram concentração e tendências. Coracini (1991) destaca a intertextualidade como uma das normas impostas pela comunidade científica, e entre os elementos que a caracterizam encontram-se as citações e referências. Segundo a autora, os dados bibliográficos, presentes no discurso científico, teriam como finalidade básica "apoiar os próprios argumentos para (...) dar respaldo, no sentido de dividir as responsabilidades quanto à metodologia" (Coracini, 1991, p. 63).

Nesse sentido, as citações bibliográficas foram utilizadas para indicar as leituras necessárias à compreensão do próprio artigo ou como forma econômica de remeter o leitor a outras pesquisas, evitando explicações demoradas sobre métodos, técnicas e teorias (Coracini, 1991). Cabe lembrar, de acordo com MacRoberts e MacRoberts (1989), que citações negativas são pouco utilizadas nos trabalhos científicos, e por esse motivo as referências bibliográficas funcionam como argumentos a favor da pesquisa em questão.

\section{Considerações finais}

A PNEPS completou dez anos desde a publicação da primeira portaria em 2014, o que justificava um estudo que permitisse sistematizar aspectos relativos à abordagem da EPS no Brasil mediante a análise de dissertações e teses publicadas em bases de dados científicas durante esse período.

O estudo permitiu observar a predominância de autores enfermeiros e assistentes sociais do gênero feminino e com filiação institucional nas regiões Sul e Sudeste do Brasil. Cabe ressaltar que a pesquisa constatou que a ausência dos termos educação permanente/educação permanente em saúde na base DeCS vem promovendo o uso de descritores (tais como educação continuada e educação em saúde) que não representam adequadamente o campo conceitual da EPS.

$\mathrm{Na}$ maioria dos trabalhos pesquisados, a EPS esteve relacionada aos temas de políticas de saúde, atenção básica e formação. Observou-se concentração de estudos do tipo descritivo e exploratório, abordagem qualitativa, com maior preferência pela entrevista semiestruturada como técnica de coleta de dados. A análise das referências bibliográficas utilizadas permitiu observar três categorias: publicações do MS, referências de autores diversos e referências relacionadas ao método adotado.

No estudo que deu origem a este artigo, a opção pela técnica bibliométrica possibilitou desvelar aspectos quantitativos do campo de conhecimento, evidenciando ângulos tais como autores, fontes de produção de conhecimento, principais autores que constituem as frentes de pesquisa no campo 
de conhecimento e outras constatações. Os dados gerados pela pesquisa também permitiram sistematizar uma face da produção científica sobre a EPS após dez anos de uma política pública dedicada à educação e à formação de trabalhadores da saúde, podendo representar insumo valioso para novos estudos nesse campo de conhecimento. Assim, sugere-se a inclusão do termo educação permanente em saúde como descritor, considerando-se a necessidade da correta identificação das publicações científicas nacionais e internacionais sobre o tema.

\section{Colaboradoras}

Eluana Borges Leitão de Figueiredo trabalhou na concepção do estudo, tabulação dos dados e redação do artigo; Mônica Villela Gouvêa e Elaine Antunes Cortez trabalharam na concepção e redação do artigo; Silvia Cristina Pereira dos Santos, Kyra Vianna Alóchio e Lauanna Malafaia da Silva Alves, na tabulação dos dados. Todas as autoras declaram que não há conflitos de interesse.

Resumen El artículo tuvo como objetivo revelar la producción del conocimiento sobre educación permanente en salud, en base a un relevamiento de tesis y disertaciones brasileñas producidas desde la publicación de la primera ordenanza relativa a la Política Nacional de Educación Permanente en 2004 hasta 2013. Se investigaron informaciones sobre autoría, contenido y hallazgos, avaluados desde la perspectiva bibliométrica. Se constató la predominancia en la autoría de mujeres con formación en enfermería y servicio social y filiación institucional en las regiones Sur y Sudeste brasileños. Se constató la concentración de estudios descriptivos-exploratorios, enfoques cualitativos y entrevistas semiestructuradas como técnica de recolección de datos. En el análisis de las referencias bibliográficas, se observaron tres categorías: publicaciones del Ministerios de la Salud, referencias de autores diversos y referencias relacionadas al método. La investigación buscó reforzar la necesidad de inclusión de la "educación permanente" como descriptor y reveló una concentración de estudios en el campo de las políticas de salud, atención básica y enseñanza.

Palabras clave educación permanente en salud; políticas públicas; bibliometría. 


\section{Notas}

1 Prefeitura de Resende, Secretaria Municipal de Saúde, Resende, Rio de Janeiro, Brasil. <eluanaoft@yahoo.com.br>

Correspondência: Estrada dos Menezes, 400, bloco Al, apartamento 802, CEP 24451-230, Colubandê, São Gonçalo, RJ, Brasil.

2 Universidade Federal Fluminense, Departamento de Planejamento em Saúde do Instituto de Saúde Coletiva, Niterói, Rio de Janeiro, Brasil.

$<$ monicagouvea@gmail.com>

3 Universidade Federal Fluminense, Escola de Enfermagem Aurora de Afonso Costa, Niterói, Rio de Janeiro, Brasil.

$<$ nanicortez@hotmail.com>

4 Prefeitura de Duque de Caxias, Duque de Caxias, Rio de Janeiro, Brasil.

<silviasantospsi@ig.com.br>

5 Universidade Estácio de Sá, Curso de Enfermagem, Macaé, Rio de Janeiro, Brasil.

$<$ kyralochio@gmail.com>

6 Instituto Federal Fluminense, Campos dos Goytacazes, Rio de Janeiro, Brasil.

<laumalafaia@gmail.com>

\section{Referências}

ALVARENGA, Lídia. Bibliometria e arqueologia do saber de Michel Foucault: traços de identidade teórico-metodológica. Ciências da Informação, Brasília, v. 27, n. 3, p. 1-9, 1998. Disponível em: <www.scielo.br/scielo.php? script $=$ sci_arttext\&pid $=$ S0100-196519980 00300002>. Acesso em: 12 out. 2016.

ARAÚJO, Carlos A. Bibliometria: evolução história e questões atuais. Em Questão, Porto Alegre, v. 12, n. 1, p. 11-32, jan.-jun. 2006.

BRASIL. Ministério da Saúde. Portaria GM/MS n. 198, 13 de fevereiro de 2004. Institui a Política Nacional de Educação Permanente em Saúde como estratégia do Sistema Único de Saúde para a formação e o desenvolvimento de trabalhadores para o setor e dá outras providências. Diário Oficial da União, Brasília, 16 fev. 2004. Seção 1. Disponível em:
$<$ www.nescon.medicina.ufmg.br/biblioteca/ imagem/1832.pdf>. Acesso em: 12 out. 2016.

BRASIL. Ministério da Saúde. Portaria GM/MS n. 1.996, 20 de agosto de 2007. Dispõe sobre as diretrizes para a implantação da Política Nacional de Educação Permanente em Saúde. Diário Oficial da União, Brasília, 22 ago. 2007. Seção 1. Disponível em: $<$ http://bvsms.saude.gov.br/bvs/saudelegis/ gm/2007/prt1996_20_08_2007.html > . Acesso em: 12 out. 2016.

BRASIL. Ministério da Educação. Relatório de Avaliação do Plano Plurianual 2008-2011. Brasília: Ministério da Educação, 2010a. Disponível em: <http://portal.mec.gov.br/ index.php? Itemid = \& gid =7 192\&option $=$ com_docman\&task=doc_download $>$. Acesso em: 12 out. 2016. 
BRASIL. Ministério da Educação. Coordenação de Aperfeiçoamento de Pessoal de Nível Superior (Capes). Plano Nacional de Pós-Graduação (PNPG) 2011-2020. Brasília: Capes, 2010b.

BRASIL. Ministério da Educação. Instituto Nacional de Estudos e Pesquisas Educacionais Anísio Teixeira. Censo da educação superior de 2012: resumo técnico. Brasília: Inep, 2013. Disponível em: <http://download. inep.gov.br/download/superior/censo/2012/ resumo_tecnico_censo_educacao_superior_ 2012.pdf > . Acesso em: 12 out. 2016.

CORACINI, Maria J. R. F. Um fazer persuasivo: o discurso subjetivo da ciência. São Paulo: Pontes, 1991.

ERDMANN, Alacoque L.; FERNANDES, Josicelia D.; TEIXEIRA, Giselle A. Panorama da educação em enfermagem no Brasil: graduação e pós-graduação. Enfermagem em Foco, Brasília, v. 2, supl., p. 89-93, 2011.

LEAL, Mariana B.; CAMARGO JUNIOR, Kenneth R. Saúde coletiva em debate: reflexões acerca de um campo em construção. Interface: Comunicação, Saúde e Educação, Botucatu, v. 16, n. 40, p. 53-65, jan.-mar. 2012.

MacROBERTS, Michael H.; MacROBERTS, Barbara R. Problems of citation analysis: a critical review. Journal of American Society for Information Science, Hoboken, v. 40, n. 5, p. 342-349, 1989.

NARIN, Francis. Evaluative bibliometrics: the use of publication and citation analysis in the evaluation of scientific activity. Washington, DC: National Science Foundation; Computer Horizons, 1976.
RODRIGUES, Rosalina A. P. et al. Pós-graduação em enfermagem no Brasil e no Nordeste. Revista Gaúcha de Enfermagem, Porto Alegre, v. 28, n. 1, p. 70-78, 2007.

SILVA, Fabiane F.; RIBEIRO, Paula R. C. A participação das mulheres na ciência: problematizações sobre as diferenças de gênero. Revista labrys, études féministes/estudos feministas, Brasília, Montreal, Paris, n. 10, p. 1-25, jul.-dez. 2011. Acesso em: 12 out. 2016.

TEIXEIRA, Elizabeth et al. Trajetória e tendências dos cursos de enfermagem no Brasil. Revista Brasileira de Enfermagem, Brasília, v. 59, n. 4, p. 479-487, 2006.

VARGAS, Eliane et al. Os usos da abordagem qualitativa na produção científica nacional recente sobre ensino em biociências e saúde. In: ENCONTRO NACIONAL DE PESQUISA E EDUCAÇÃO EM CIÊNCIA, 8, Florianópolis, 2009. Florianópolis: Associação Brasileira de Pesquisa em Educação em Ciências, 2009. Disponível em: <http:// posgrad.fae.ufmg.br/posgrad/viienpec/pdfs/ 1354.pdf $>$. Acesso em: 12 de outubro de 2016.

VIEIRA, Ana L. S. et al. Tendências do sistema educativo no Brasil: medicina, enfermagem e odontologia. In: BARROS, André F. R. (org.). Observatório de recursos humanos em saúde no Brasil: estudos e análises. Brasília: Ministério da Saúde, 2004. v. 2. p. 183-202.

Recebido em 10/10/2014

Aprovado em 13/04/2015 